\title{
Dominant negative mutant Cyclin TI proteins inhibit HIV transcription by specifically degrading Tat
}

\author{
Julie K Jadlowsky¹, Masanori Nojima ${ }^{1}$, Antje Schulte ${ }^{2}$, Matthias Geyer ${ }^{2}$, \\ Takashi Okamoto ${ }^{3}$ and Koh Fujinaga*1
}

\begin{abstract}
Address: ${ }^{1}$ Division of Infectious Diseases, Department of Medicine, Department of Molecular Biology and Microbiology, Case Western Reserve University School of Medicine, Cleveland, Ohio, USA, ${ }^{2}$ Max-Planck-Institut für molekulare Physiologie, Abteilung Physikalische Biochemie, Dortmund, Germany and ${ }^{3}$ Department of Molecular and Cellular Biology, Nagoya City University Graduate School of Medical Sciences, Nagoya, Japan

Email: Julie K Jadlowsky - julie.jadlowsky@case.edu; Masanori Nojima - nojima@sapmed.ac.jp; Antje Schulte - antje.schulte@mpidortmund.mpg.de; Matthias Geyer - matthias.geyer@mpi-dortmund.mpg.de; Takashi Okamoto - tokamoto@med.nagoya-cu.ac.jp; Koh Fujinaga* - kxf32@cwru.edu

* Corresponding author
\end{abstract}

Published: II July 2008

Retrovirology 2008, 5:63 doi:10.1 186/1742-4690-5-63

This article is available from: http://www.retrovirology.com/content/5/I/63

(C) 2008 Jadlowsky et al; licensee BioMed Central Ltd.

This is an Open Access article distributed under the terms of the Creative Commons Attribution License (http://creativecommons.org/licenses/by/2.0), which permits unrestricted use, distribution, and reproduction in any medium, provided the original work is properly cited.
Received: 9 April 2008

Accepted: II July 2008

\begin{abstract}
Background: The positive transcription elongation factor b (P-TEFb) is an essential cellular cofactor for the transcription of the human immunodeficiency virus type I (HIV-I). The cyclin TI (CycTI) subunit of P-TEFb associates with a viral protein, Tat, at the transactivation response element (TAR). This represents a critical and necessary step for the stimulation of transcriptional elongation. Therefore, CycTI may serve as a potential target for the development of anti-HIV therapies.
\end{abstract}

Results: To create effective inhibitors of HIV transcription, mutant CycTI proteins were constructed based upon sequence similarities between CycTI and other cyclin molecules, as well as the defined crystal structure of CycTI. One of these mutants, termed CycTI-U7, showed a potent dominant negative effect on Tat-dependent HIV transcription despite a remarkably low steady-state expression level. Surprisingly, the expression levels of Tat proteins co-expressed with CycTI-U7 were significantly lower than Tat co-expressed with wild type CycTI. However, the expression levels of CycTI-U7 and Tat were restored by treatment with proteasome inhibitors. Concomitantly, the dominant negative effect of CycTI-U7 was abolished by these inhibitors.

Conclusion: These results suggest that CycTI-U7 inhibits HIV transcription by promoting a rapid degradation of Tat. These mutant CycTI proteins represent a novel class of specific inhibitors for HIV transcription that could potentially be used in the design of anti-viral therapy.

\section{Background}

The transcription of human immunodeficiency virus type 1 (HIV-1) is a highly regulated process in which several host cellular co-factors and the viral transactivator protein Tat are involved $[1,2]$. Tat stimulates the elongation of transcription with the aid of the positive transcription elongation factor $\mathrm{b}(\mathrm{P}-\mathrm{TEFb})$, a heterodimer comprised of cyclin T1 (CycT1) and cyclin dependent kinase 9 (Cdk9). Tat and CycT1 bind to the transactivation response element (TAR), an RNA stem loop structure located at the 5'- 
end $(+1$ to +59$)$ of all viral transcripts [3-5]. This interaction results in the recruitment of $\mathrm{Cdk} 9$ and the subsequent stimulation of its kinase activity by Tat [6]. Among three distinct P-TEFb complexes (CycT1/Cdk9, CycT2/ Cdk9, and CycK/Cdk9), only the CycT1/Cdk9 complex can support Tat transactivation [7-9].

The interaction between Tat, TAR, and CycT1 has been extensively studied $[2-5,8,10]$. Tat binds to the bulge region $(+23$ to +25$)$ of TAR and the CycT1 subunit of P$\mathrm{TEFb}$ through its central arginine-rich motif (ARM; a.a. 49-60) and its N-terminal activation domain (a.a. 1-48), respectively. CycT1, in turn, is thought to bind to the central loop (+30 to +35$)$ of TAR through its Tat-TAR recognition motif (TRM; a.a. 251-271) in the presence of Tat $[1,2]$. Human CycT1 is comprised of 726 amino acids and contains a cyclin box repeat domain (from positions 31 to 250), a coiled-coil sequence (from positions 379 to 530), and a PEST sequence (from positions 709 to 726). The $\mathrm{N}-$ terminal cyclin boxes are important for binding and activation of Cdk9. Residues from positions 251 to 272 are essential for the zinc ion-mediated binding between Tat and TAR [5]. This region also interacts with the HEXIM1 protein and 7SK small nuclear RNA, which negatively regulate the kinase activity of P-TEFb [11-15]. The C-terminal region (a.a. 273-726) of CycT1 is dispensable for Tat transactivation since the $\mathrm{N}$-terminal cyclin repeats (a.a. 1250) and TRM (a.a. 251-272) of CycT1 interact with Cdk9, Tat and TAR $[3-5,9,16,17]$. Recently, we have determined the crystal structure of the $\mathrm{N}$-terminal region (a.a. 1-280) of human CycT1 [18] and its interacting dimeric Cyclin T-binding domain in HEXIM1 [19].

Since P-TEFb is the essential cellular host co-factor of the viral Tat protein, this interaction serves as a potential target for anti-HIV therapeutics. Several approaches have been taken to block HIV transcription by targeting P-TEFb. First, mutant Cdk9 proteins defective in kinase activity have been shown to inhibit HIV transcription in cell culture systems [20]. A number of small compounds that inhibit Cdk9 activities or disrupt the Tat/TAR/P-TEFb interaction have also been tested [20-28]. Another approach by Napolitano et al. aimed to inactivate Cdk9 by an oligomerization chain reaction [29]. Additionally, our group has constructed chimeric proteins containing wild type (wt) CycT1 and mutant Cdk9 which inhibited HIV replication up to $90 \%$ [30]. Moreover, several CycT1binding proteins and their truncation mutants have been used as inhibitors of Tat transactivation [31-33]. Finally, Bai et al. demonstrated that intrabodies against CycT1 inhibited Tat stimulated transactivation [34]. It is important to note, however, that because P-TEFb is involved in the transcription of many cellular genes [35], it is critical to exclusively block HIV-specific pathways in order to develop safe and effective anti-HIV therapies.
In this study, we sought to construct dominant negative CycT1 mutant proteins capable of blocking HIV transcription. A sequence alignment between the cyclin proteins CycT1, T2 and $\mathrm{K}$ revealed ten very well-conserved regions that are essential for the formation of the alpha-helical cyclin box repeat domain. We introduced random mutations in the nine most conserved amino acid clusters in these regions and tested the resulting mutant CycT1 proteins for their ability to block HIV transcription. One of the mutant proteins, called CycT1-U7, showed a potent, yet specific, dominant negative effect on HIV transcription, although the steady-state expression level of CycT1U7 was remarkably low. Western blot analysis indicated that the expression level of the Tat proteins co-expressed with CycT1-U7 was also significantly lower than those coexpressed with wt CycT1. Proteasome inhibitors restored the expression of CycT1-U7 and Tat proteins. As a consequence, these inhibitors diminished the dominant negative effect elicited by over-expression of CycT1-U7. Our results suggest that CycT1-U7 inhibits HIV transcription by promoting a rapid degradation of Tat proteins. These mutant CycT1 proteins represent a novel class of specific inhibitors for HIV transcription, which might be further utilized in development of safe and effective anti-HIV therapies.

\section{Results \\ Construction and screening of CycTI mutants}

CycT1 is a member of the C-type cyclin family [36]. Its Nterminal 250 amino acids form two cyclin repeat boxes that are essential for the interaction with, and the activation of, Cdk9. Recently, we have determined the three dimensional crystal structure of CycT1 [18]. The cyclin boxes consist of two repeats, each containing five $\alpha$-helices (Figure. 1A and 1B). Sequence alignment of three PTEFb-forming cyclins $\mathrm{T} 1, \mathrm{~T} 2$, and $\mathrm{K}$ from different species revealed that the secondary structure elements are well conserved among these cyclins, indicating that they play important roles in P-TEFb functions (Figure. 1B). Based on this secondary structure alignment, we selected the nine most conserved amino acid clusters in the cyclin box domain of CycT1 and introduced random mutations into a C-terminal truncation mutant of CycT1 (CycT1(1280)). This truncation is sufficient to support Tat transactivation as described before $[4,5,9]$ (Figure. 1C and Table $1)$.

Mutations were introduced by oligonucleotides containing degenerate nucleotides corresponding to each conserved region. In total, 115 CycT1 mutants were constructed and tested for their activities on Tat transactivation by co-transfecting murine NIH 3T3 cells with an HIV LTR-Luciferase (Luc) reporter gene and Tat (Table 1). Since murine endogenous CycT1 (mCycT1) cannot support Tat transactivation, Tat activated the LTR-driven Luc 


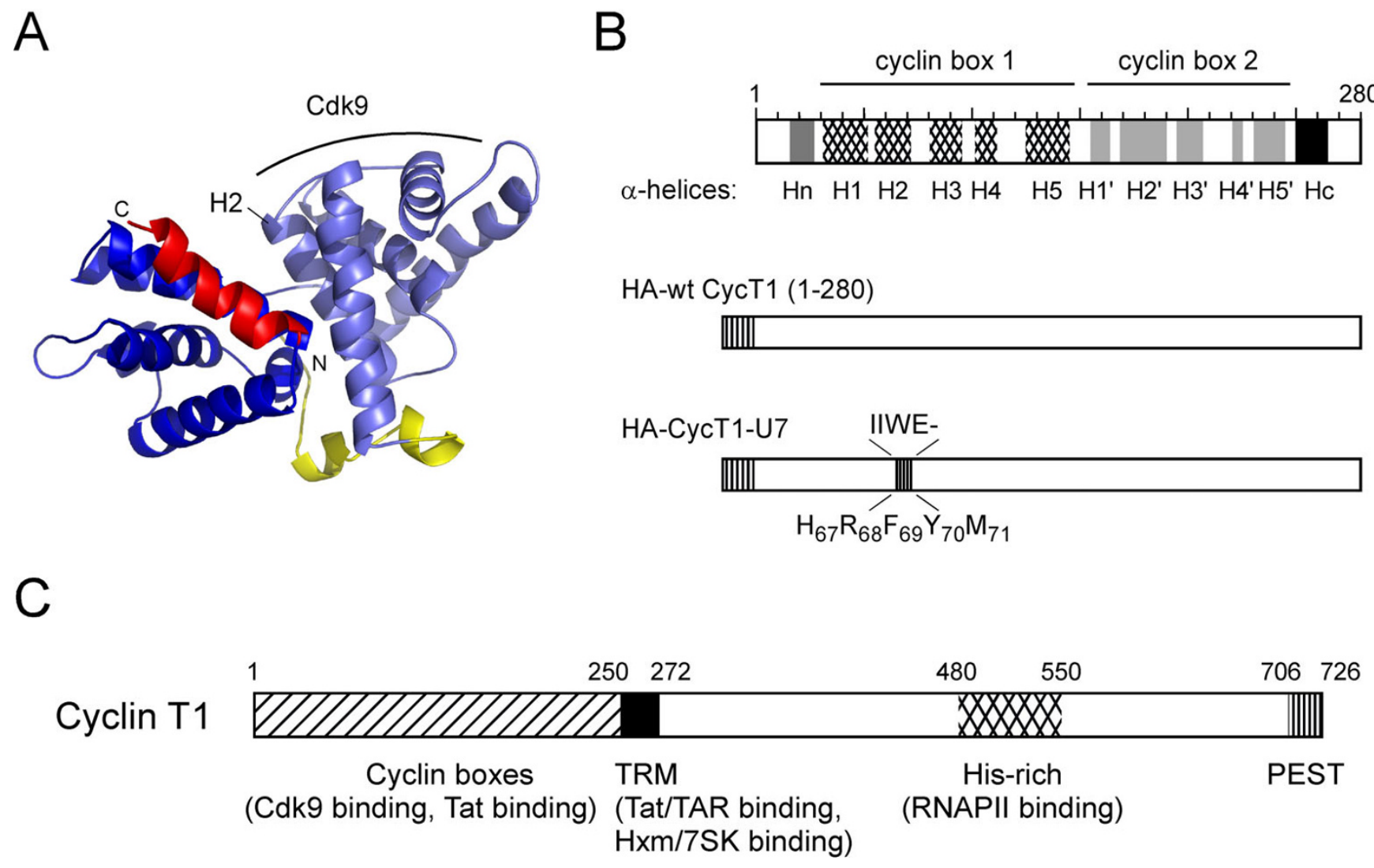

Figure I

Construction of mutant CycTI proteins.A. Structure of the cyclin box repeat domain (I-28I) of CycTI. Two repeats of five $\alpha$-helices each form the conserved cyclin box (blue). Flanking $\mathrm{N}$ - and $\mathrm{C}$-terminal helices, which are important for the specificity of cyclins, are depicted in yellow and red, respectively. B. Schematic representation of C-terminally truncated wt CycT I and the dominant negative CycTI-U7 mutant used in this study. Secondary structure of conserved $\alpha$-helices (dotted regions in cyclin box I and hatched regions in cyclin box 2) together with two helices at $\mathrm{N}$ - and C-terminal (gray) locate in the $\mathrm{N}$-terminal cyclin boxes in CycTI. Random mutations were introduced into the nine most conserved regions (shown by thin lines) in the cyclin box domain of a C-terminal truncation mutant of CycTI (CycTI(I-280)). "-" in the CycTI-U7 sequence represents a deletion site. The truncated wt and mutant CycTI employed in this study are also shown. C. A schematic representation of the full-length Cyclin TI. Amino acid motifs such as cyclin boxes, Tat-TAR recognition motif (TRM), coiled-coiled region, and PEST sequence are depicted.

expression only by approximately 10-fold (Figure. 2A, lane 2). Over-expression of the wt human CycT1 further activated the gene expression up to 70-fold (Figure. 2A, lanes 3 and 4). The luciferase activities obtained by overexpressing any of the pool of mutant CycT1 proteins ranged from five to 70 -fold. Fifteen mutants showed an equal or a higher activity than the wt, 45 mutants showed modest (50-100\% of wt) activity and 55 had less than $50 \%$ of the activity of wt CycT1 in these cells (summarized in Table 1). These 55 mutants were further sequenced and tested for their dominant negative effect on HIV transcription by co-transfecting HeLa cells stably expressing the HIV-Luc reporter gene (HeLa/HR-Luc cells) with Tat (Figure. $2 \mathrm{~B}$ and data not shown).
An N-Terminal CycTI mutant exhibited the strongest dominant negative effect on Tat transactivation by promoting the degradation of Tat proteins

Amongst the 55 clones tested for their ability to block Tat transactivation in HeLa cells, one mutant containing four amino acid substitutions and one deletion in the second helix $\mathrm{H} 2$ of the $\mathrm{N}$-terminal cyclin box repeat (residues HRFYM at a.a. position 67-71 to IIWE; Figure. 1B), termed CycT1-U7, showed the strongest dominant negative effect ( $>90 \%$ inhibition) on HIV transcription in HeLa/HR-Luc cells (Figure. 2B, lanes 3 to 5). At least four other mutant CycT1 constructed by the same oligonucleotides (Mut 2, Additional file 1) showed potent dominant negative effects on HIV transcription (60-90\%, data not 
Table I: Overview of CycT I mutants used in this study.

\begin{tabular}{|c|c|c|c|c|c|c|}
\hline \multirow[t]{2}{*}{ Regions } & \multirow[t]{2}{*}{ Helix\# } & \multirow[t]{2}{*}{ Amino acid positions } & \multirow[t]{2}{*}{ \# of clones } & \multicolumn{3}{|c|}{ Activity in murine cells $(* I)$} \\
\hline & & & & $>100 \%$ & $50-100 \%$ & $<50 \%$ \\
\hline Region I & 2 & $58-65$ & 29 & 4 & 14 & 11 \\
\hline Region 2 & 2 & $67-71$ & 26 & 3 & 10 & 13 \\
\hline Region 3 & 3 & $88-91$ & 16 & 2 & 5 & 9 \\
\hline Region 4 & 3 & $93-96$ & 16 & 3 & 11 & 2 \\
\hline Region 5 & 4 & $104-108$ & 5 & 0 & 0 & 5 \\
\hline Region 6 & 5 & $132-137$ & 3 & 0 & I & 2 \\
\hline Region 7 & 5 & $139-143$ & 9 & 0 & 0 & 9 \\
\hline Region 8 & 5 & $145-148$ & 2 & 0 & 0 & 2 \\
\hline Region 9 & $I^{\prime}$ & $149-152$ & 9 & 3 & 4 & 2 \\
\hline total & & & 115 & 15 & 45 & 55 \\
\hline
\end{tabular}

*I: Tat transactivation obtained with wt human CycTI is set as 100\%

shown). Over-expression of CycT1-U7 affected neither the basal HIV transcription nor CMV-Luc reporter gene expression (Figure. 2C). Next, HeLa/HR-Luc cells stably expressing CycT1-U7 were created by infecting with a second lentiviral vector. Tat transactivation in these cells was scored by transfecting an increasing amount of Tat and measuring LTR-driven luciferase activity. In cells expressing CycT1-U7, Tat exhibited a significantly lower activity compared to the cells stably carrying the empty pHR lentiviral vector (Figure. 2D). Western blot analysis revealed that the steady-state expression level of CycT1-U7 is much lower than wt CycT1 (1-280) although the same amount of plasmid was transfected (Figure. 3A, lanes 2 and 3 in the top panel). Interestingly, the expression level of Tat was also much lower in CycT1-U7-expressing cells than in wt CycT1 (1-280) expressing cells, (Figure. 3A, lane 2 and $3)$. In contrast, the expression levels of the endogenous CycT1 and Cdk9 in the presence of CycT1-U7 remained unchanged (Figure. 3A). These results suggested that Tat transactivation in CycT1-U7 expressing cells is kept at a low level because the steady state Tat expression is diminished in these cells. Since CycT1-U7 retains the wild type sequence of Tat-TAR recognition motif (Figure. 1C), we hypothesized that CycT1-U7 forms a complex with Tat, and this complex is rapidly degraded in cells.

\section{Expression of CycTI-U7 and Tat can be rescued by proteasome inhibitors}

To further prove our hypothesis that CycT1-U7, together with Tat, is rapidly transferred to proteasomal degradation pathways, cells expressing Tat and either wt CycT1 (1280) or mutant CycT1-U7 were incubated with the proteasome inhibitors, MG-132 $(50 \mu \mathrm{M})$ or Epoxomicin (50 $\mu \mathrm{M}$ ) for 1,3 , and 5 hours prior to cell lysis. MG-132 showed a strong cytopathic effect when incubated for 5 hours (data not shown). The expression of both CycT1-U7 and Tat was partially restored in the presence of MG-132
(Figure. 3B, lanes 2 and 3 compared with lane 1), and much more efficiently restored in the presence of Epoxomicin (Figure. 3B, lanes 5 to 7 compared with lane 4). In contrast, the expression of wt CycT1 (1-280) and Tat remained virtually unchanged in the presence of these inhibitors (lanes 9 and 10 compared with lane 8).

The restoration of the CycT1-U7 and Tat expression by Epoxomicin was also observed at the cellular level by an indirect immuno-fluorescence (IF) assay (Figure. 4A). HAtagged wt and mutant CycT1 and myc-tagged Tat proteins were co-expressed in HeLa/HR-Luc cells. Twenty-four hours after transfection, cells were untreated or treated with $25 \mu \mathrm{M}$ Epoxomicin for 3 hours. HA-CycT1 proteins were probed with mouse anti-HA and Cy2-conjugated anti-mouse IgG, and myc-Tat proteins were probed with Texas Red-labelled anti-myc antibody. As shown in Figure. 4A, the expression of CycT1-U7 and Tat was kept at low levels without Epoxomicin treatment. The protein levels were elevated when the cells were treated with Epoxomicin. The wt CycT1 and Tat proteins co-expressed with wt CycT1 were detected in the presence or absence of Epoxomicin. Finally, the inhibitory effect by CycT-U7 was diminished in transient (Figure. 4B) and stable (Figure. 4C) expression systems when the cells were incubated with $25 \mu \mathrm{M}$ Epoxomicin for 6 to 18 hours. Since it has been demonstrated that CycT1 is ubiquitinated in cells [37], we sought to examine whether CycT1-U7 is ubiquitinated by co-immunoprecipitation analysis (Figure. 5A). Ubiquitinated CycT1-U7 proteins were detected in HeLa/ CycT1-U7 cells treated with $50 \mu \mathrm{M}$ Epoxomicin for 60 min (Figure. 5A, lane 2). Also, in this condition, the interaction between CycT1-U7 and Tat was detected by coimmunoprecipitation (Figure. 5B, lane 4). These results suggest that CycT1-U7 inhibits Tat-transactivation by rapidly recruiting Tat proteins into an ubiquitin-dependent proteasomal degradation pathway. 


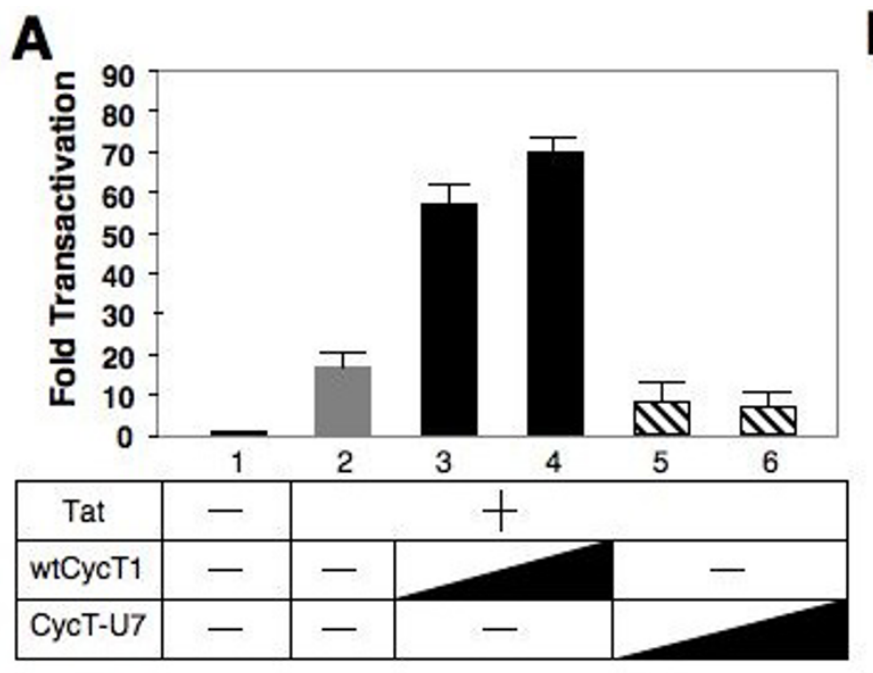

B
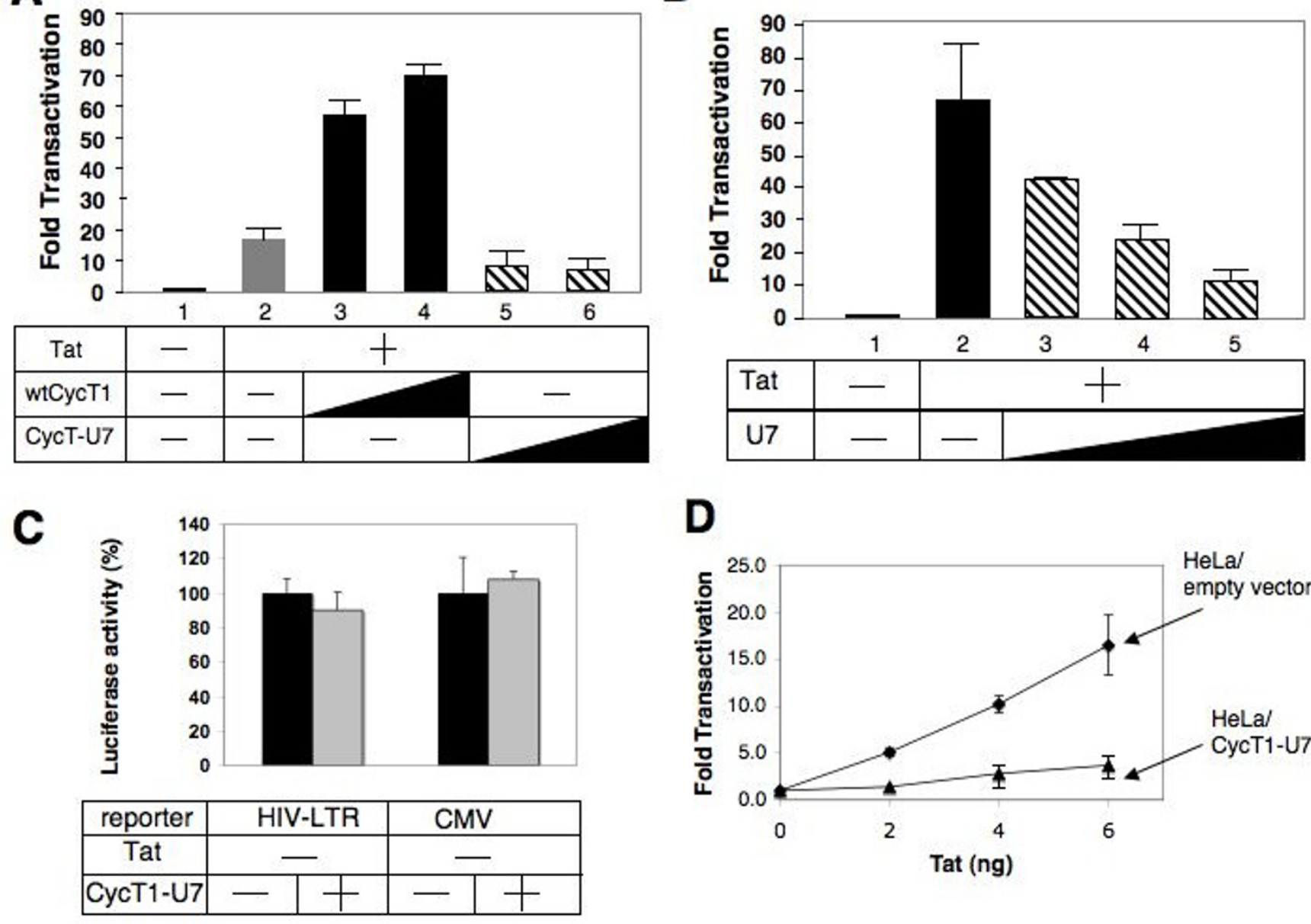

D

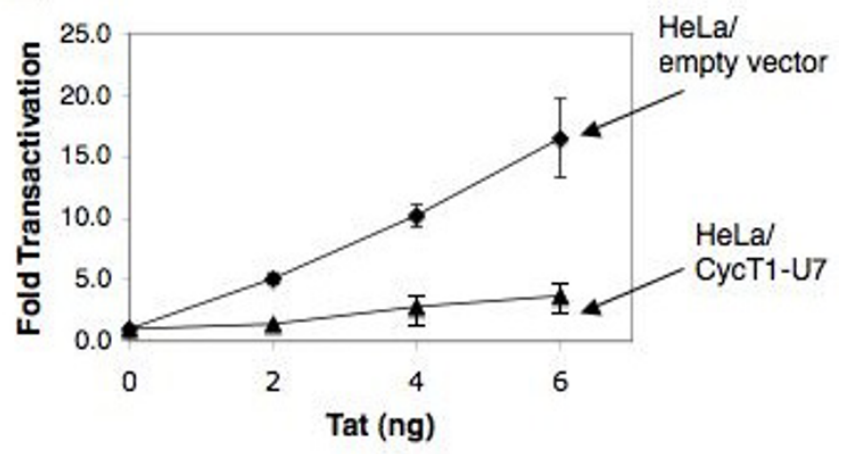

Figure 2

N-terminal mutant CycTI proteins (CycTI-U7) exhibit a strong dominant negative effect on HIV transactivation.A. CycTI-U7 cannot support Tat transactivation in murine cells. NIH 3T3 cells were transfected with HIV-Luc reporter gene in the presence (lane 2-6) or absence (lane I) of Tat $(0.1 \mu \mathrm{g})$ with or without increasing amounts $(0.2$ and $0.5 \mu \mathrm{g})$ of wt human CycT I-280 (lanes 3 and 4) or CycTI-U7 (lanes 5 and 6). Twenty-four hours after transfection, luciferase activities were measured as described before. B. CycTI-U7 shows strong dominant negative effects on Tat-transactivation. Increasing amounts of CycTI-U7 (0.2, 0.4 and $0.6 \mu \mathrm{g})$ were transfected in HeLa/pHR-Luc cells in the presence of Tat $(0.02 \mu \mathrm{g})$. Luciferase activities were measured as described above. C. CycTI-U7 was unable to inhibit basal HIV transcription and CMV-driven transcription. The plasmid $(0.6 \mu \mathrm{g})$ encoding CycTI-U7 (gray bars) or an empty vector (black bars) was co-transfected in HeLa cells with HIV-LTR-Luciferase or CMV-Luciferase reporter plasmid $(0.05 \mu \mathrm{g})$ in the absence of Tat. Luciferase activity was measured as described above. D. Tat has lower activity on HIV-LTR in cells stably expressing CycTI-U7. Increasing amounts of Tat were transfected in Hela/pHR-Luc cells stably carrying a lentiviral vector encoding no protein (empty vector; gray diamonds) or CycTI-U7 proteins (black triangle). Luciferase activities were measured as described in the Materials and Methods section. Error bars represent the standard deviation of triplicate measurements. Data are representative of four independent assays.

\section{Discussion}

Although P-TEFb is a potential target for the development of novel anti-HIV therapies, it had been extremely difficult to construct dominant negative CycT1 mutants that block HIV transcription [30]. This is presumably due to the high stability and the complex regulatory mechanism of the endogenous P-TEFb complex. In the present study, we constructed and evaluated a novel class of CycT1 mutant proteins (CycT1-U7) that explicitly block HIV transcription by promoting a rapid and specific degradation of Tat proteins co-expressing CycT1-U7. Resulting from a functional screen of 115 randomized mutant proteins, sequence analysis of CycT1-U7 showed five mutations including one amino acid deletion in the second helix of 


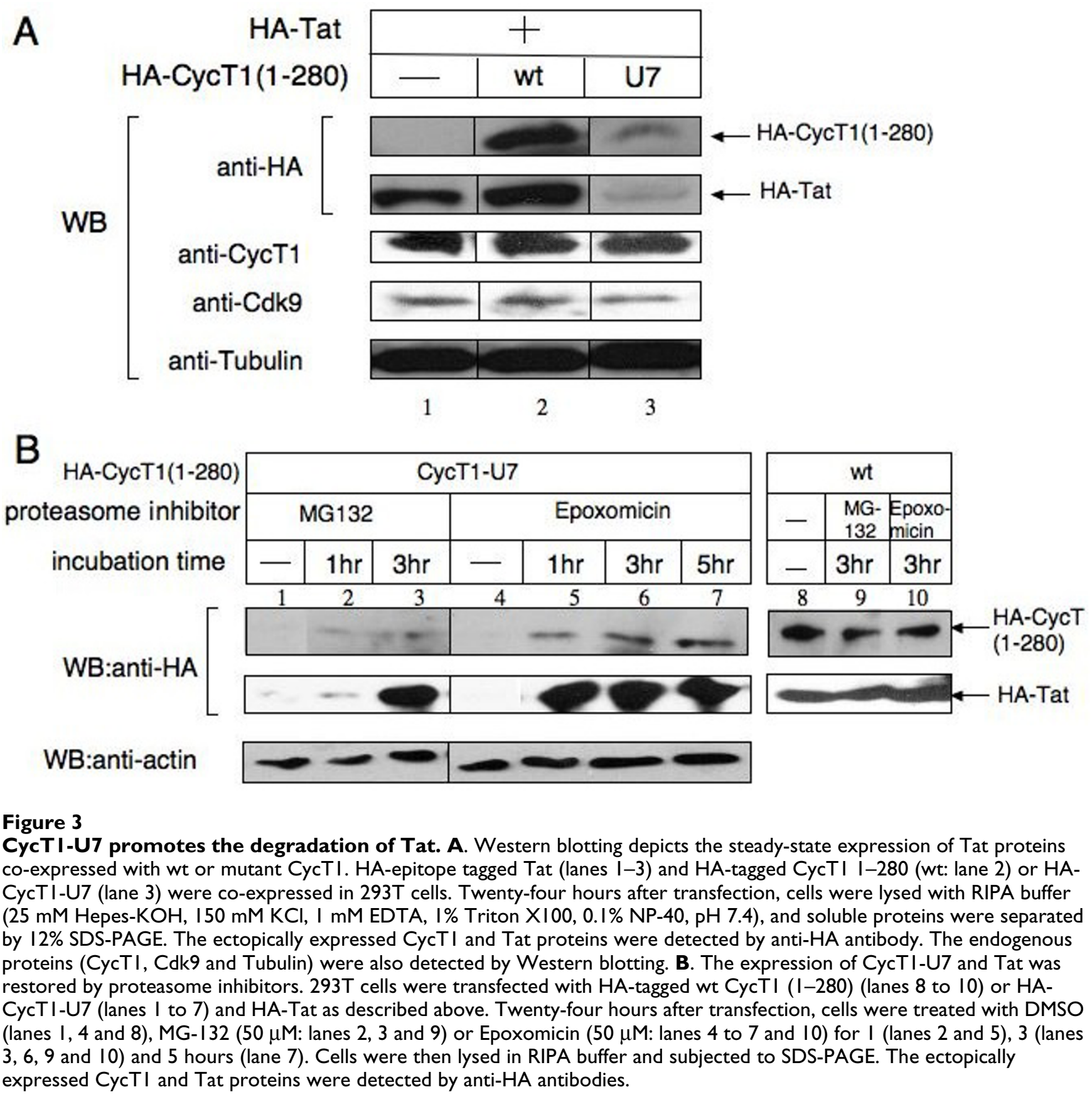

the cyclin box repeat fold (Figure. 1B). We have previously demonstrated that a CycT1 variant lacking this region (CycT1 (119-280)) is also unstable in cells [38]. This particular mutant exhibited a potent dominant negative effect on HIV transcription, potentially by a similar mechanism (data not shown). Therefore, H2 of CycT1 appears very important for maintaining the structural stability of CycT1 and the interface in between the two repeats. In addition, a residue directly preceding the first helix of the cyclin box repeat that varies between human and equine
CycT1 has been previously identified as responsible for differences in the recognition of Tat/TAR complexes from HIV and EIAV [39]. Together, these data point towards the importance of the integrity of the first cyclin box repeat for the interaction with Tat. This region also appears to be essential for the interaction with Cdk9 $[18,30]$. Interestingly, CycT1-U7 does not promote degradation of endogenous Cdk9. On the other hand, this mutant does bear the wild type sequence of Tat/TAR recognition motif (a.a. 251-272). Indeed, the complex between CycT1-U7 and 

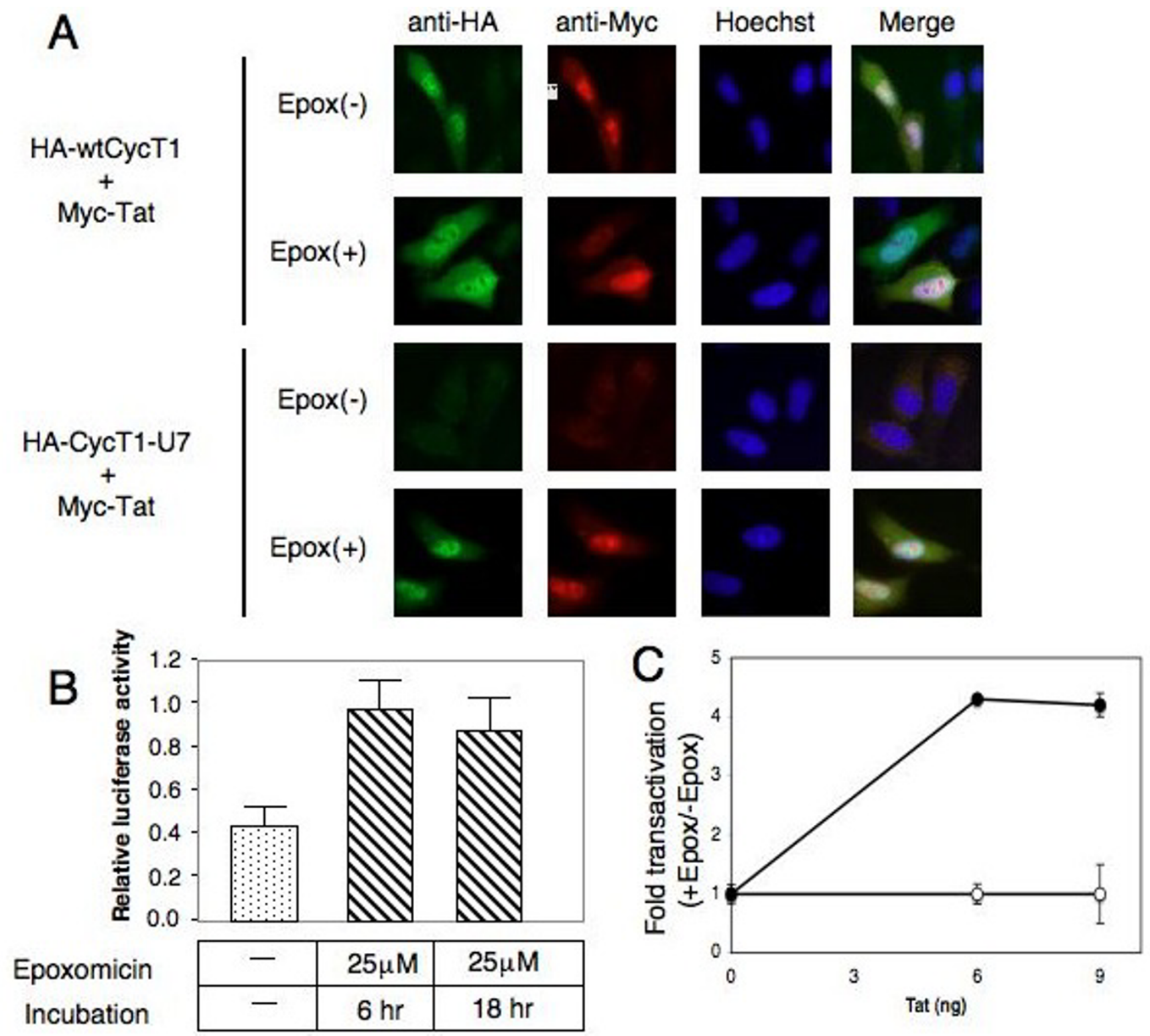

Figure 4

Epoxomicin restores CycTI-U7 and Tat expression.A. HA-tagged wt and mutant CycTI and myc-tagged Tat proteins were co-expressed in HeLa/HR-Luc cells. Twenty-four hours after transfection, cells were untreated or treated with $25 \mu \mathrm{M}$ Epoxomicin for 3 hours. HA-CycTI proteins were visualized with mouse anti-HA antibody and Cy2-conjugated donkey antimouse lgG. Myc-Tat proteins were seen with Texas Red-conjugated anti-myc antibody. Nuclei were stained with Hoechst. B. The inhibitory effect by CycTI-U7 was diminished by Epoxomicin. HeLa/HR-Luc cells were transfected with CycTI-U7 expression plasmids $(0.5 \mu \mathrm{g})$ or empty plasmids $(0.5 \mu \mathrm{g})$ in the presence of Tat. Cells were treated with DMSO (-) or $25 \mu \mathrm{M}$ Epoxomicin for 6 hours and 18 hours as indicated, and the Luc activities were measured. The results were presented as relative luciferase values obtained with CycTI-U7 divided by the values with the empty vector at each time point. C. Increasing amounts of Tat were transfected in Hela/pHR-Luc cells stably expressing CycTI-U7 proteins. 24 hours after transfection, cells were untreated (open circles) or incubated (closed circles) with Epoxomicin $(25 \mu \mathrm{M})$ for 6 hours prior to luciferase assay. Data are presented as fold activation relative to the value obtained with untreated cells. Error bars represent the standard deviation of triplicate measurements. Data are representative of three independent assays. 


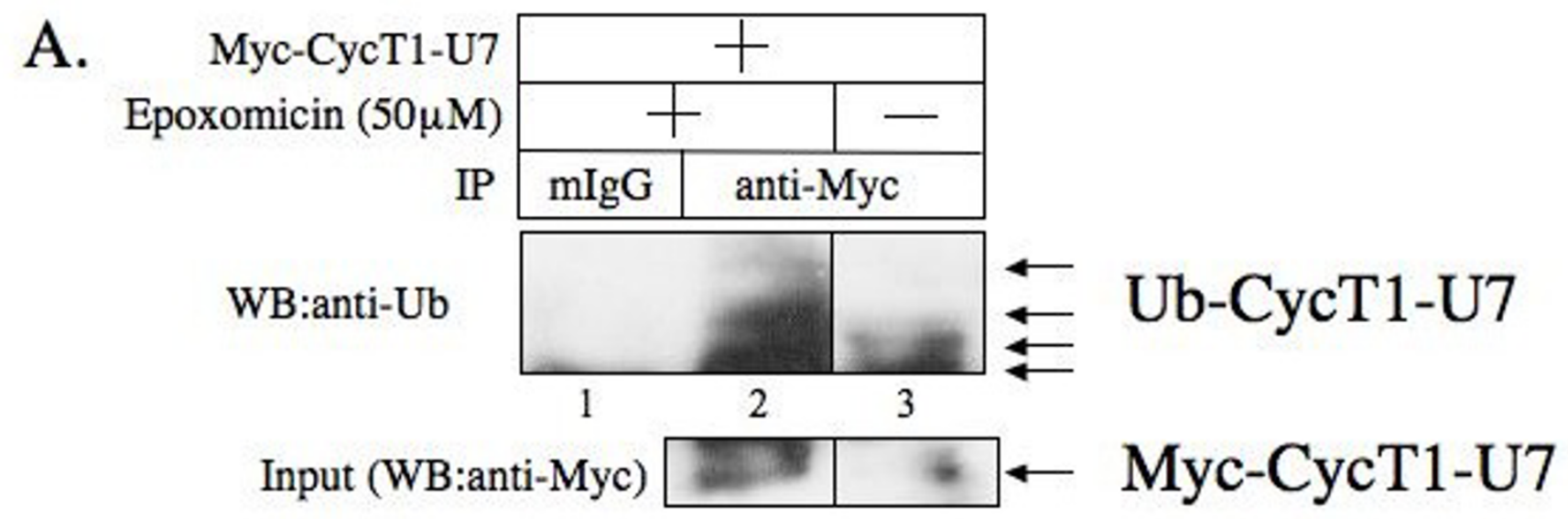

B.

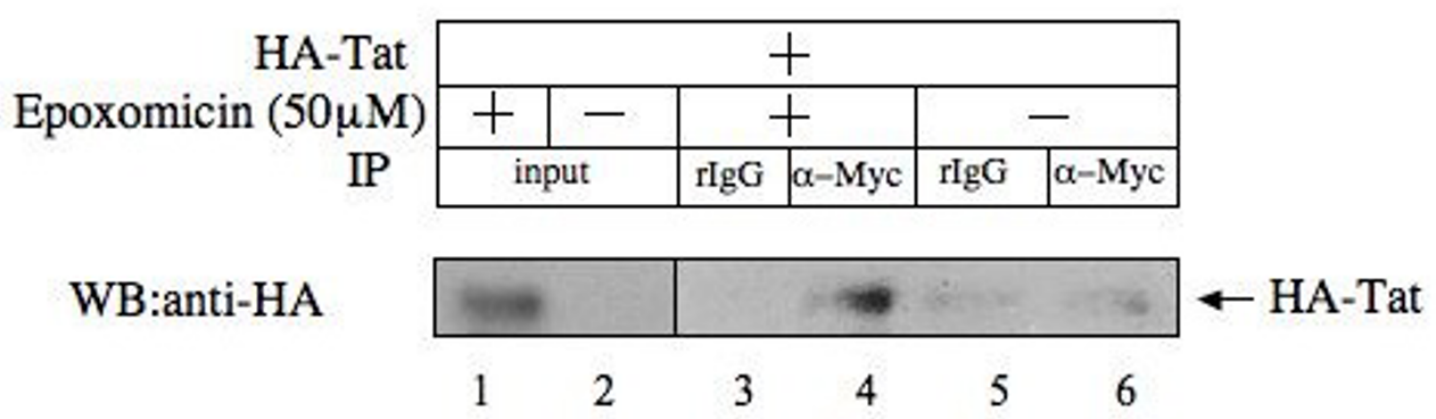

\section{Figure 5}

CycTI-U7 is ubiquitinated.A. HeLa cells stably expressing myc-CycTI-U7 proteins (HeLa/myc-CycTI-U7) were treated (lanes I and 2) or untreated (lane 3) with Epoxomicin (50 $\mu \mathrm{M})$ for 30 min prior to cell lysis. The Myc-CycTI-U7 proteins were immunoprecipitated with anti-Myc antibody followed by Western blot analysis with anti-Ub antibody to detect ubiquitinated Myc-CycTI-U7 proteins (upper panel). Normal mouse lgG (mlgG) was used as a negative control for immunoprecipitation (lane I). The expression of the Myc-CycTI-U7 proteins in 10\% of the input samples was also detected by Western blot analysis using anti-Myc antibody (lower panel). B. CycTI-U7 binds Tat. HeLa/Myc-CycTI-U7 cells were transfected with HA-Tat. The cells were treated (lanes I, 3 and 4) or untreated (lanes 2, 4 and 5) with Epoxomicin (50 $\mu$ M) for 30 min prior to cell lysis. The Myc-CycTI-U7 proteins were immunoprecipitated with anti-Myc antibody (lanes 4 and 6) followed by Western blot analysis with anti-HA antibody to detect Tat proteins. Normal rabbit lgG (rlgG) was used as a negative control (lanes 3 and 5). Tat proteins in the input samples (I0\%) were also shown (lanes I and 2).

Tat was detected when the cells were treated with proteasome inhibitors (Figure. 5). The mutant CycT1-U7 proteins can form a complex with Tat and this complex would be immediately degraded because of the instability of CycT1U7. Therefore, we conclude that CycT1-U7 exhibits a strong dominant negative effect on Tat transactivation by specifically degrading the co-expressed Tat protein, without disturbing the endogenous P-TEFb complex (Figure. $6)$.

It has been demonstrated that CycT1 interacts with other cellular transcription factors through its $\mathrm{N}$-terminal cyclin box regions [40,41]. It is of importance to examine whether CycT-U7 can also inhibit cellular transcription mediated by these factors via a similar pathway. Additionally, the TRM region of CycT1 also interacts with HEXIM1, the endogenous inhibitory protein of $\mathrm{P}-\mathrm{TEFb}$ which interacts with this region [11], and it is possible that CycT1-U7 affects P-TEFb activity by reducing HEXIM1 levels. More detailed studies are required to assess the effect of CycT1U7 on cellular transcription.

Our results indicate that CycT1-U7/Tat is recruited to the ubiquitin-dependent degradation pathway. CycT1 seems to be ubiquitinated not only on its C-terminal PEST region (a.a. 706-726) but also at other regions [37]. It is to be noted that wt CycT1 (1-280) is resistant to degradation (Figure. 3). Although we have not identified the 


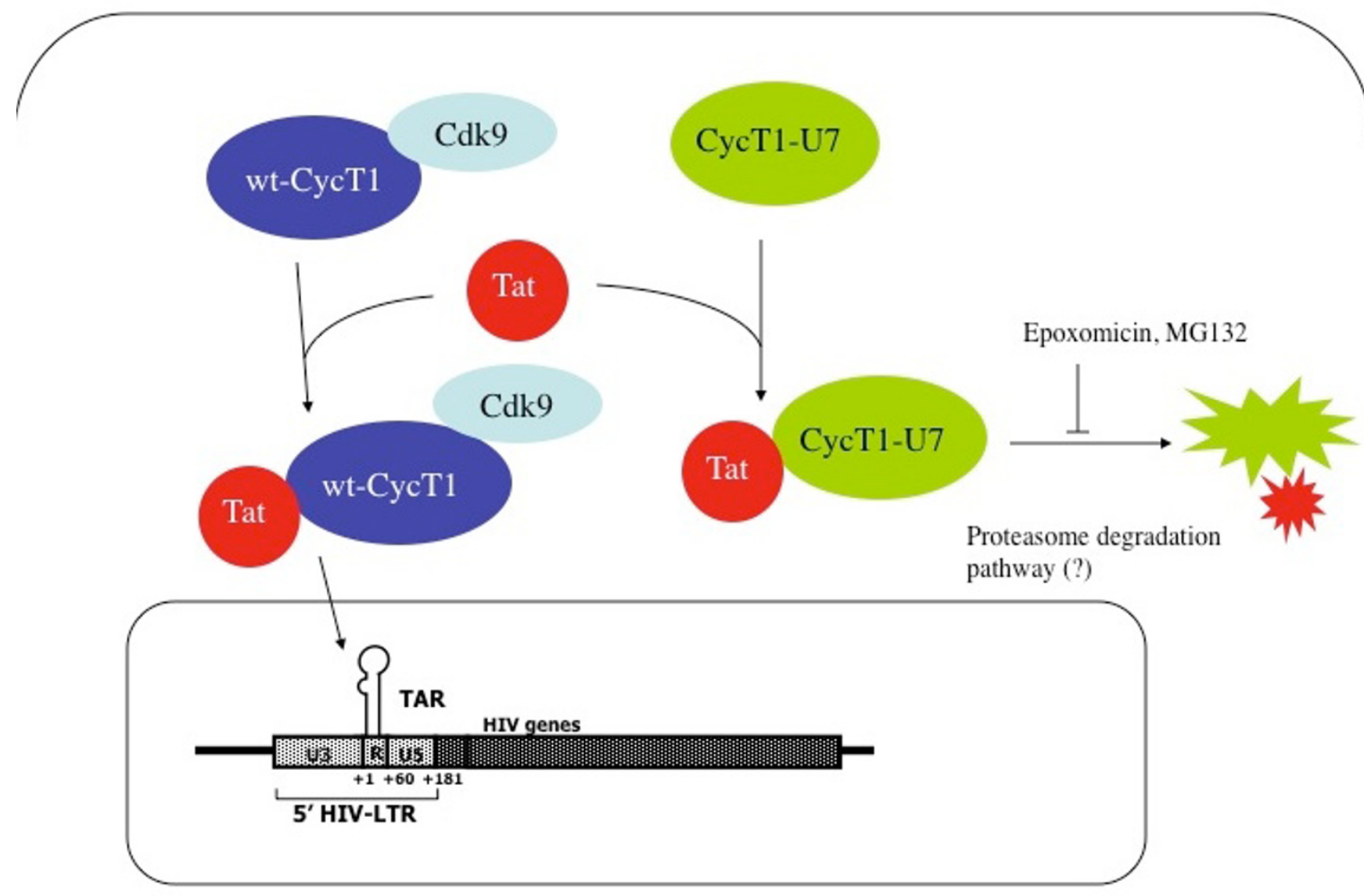

Figure 6

Proposed model for the mechanism of dominant negative effect elicited by CycTI-U7. Wild type CycTI forms a complex with Cdk9 as an active P-TEFb, and interacts with Tat and TAR RNA. Alternatively, CycTI-U7 associates with Tat but the CycT-U7/Tat complex is immediately degraded via an ubiquitin-dependent proteasomal pathway. This degradation can be prevented using proteasome inhibitors.

potential ubiquitination site(s) of CycT1-U7 in this study, it is possible that the cyclin box structure stabilizes the protein by preventing ubiquitination. Conformational changes induced by post-translational modifications such as phosphorylation may expose any additional ubiquitination sites in this region, which would represent a novel pathway to regulate P-TEFb function.

Constructing CycT1 mutants based on C-terminal truncated forms of wt CycT1 (CycT1(1-280)) is particularly beneficial in terms of HIV transcription. CycT1 (1-280) has been demonstrated to be sufficient for supporting Tattransactivation $[4,5,9]$. In addition, Tat competes with HEXIM1 to increase active P-TEFb complexes $[14,15,19]$. CycT1 (1-280) can therefore bypass the 7SK/HEXIMmediated complex regulatory pathway and be exclusively directed towards Tat-dependent transactivation (Jad- lowsky et al., unpublished data), making CycT1 (1-280) proteins highly specific for Tat.

Since the mechanism by which CycT1-U7 inhibits HIV transcription seems not to be through blocking the normal function of P-TEFb, but rather through a "gain-offunction" pathway, it represents a novel class of inhibitory molecules. Moreover, since the steady-state expression of CycT-U7 is very low, it may be an excellent candidate for gene therapy because the mutant proteins would not persist for a prolonged period of time, thereby avoiding induction of unwanted immune responses. Additionally, these proteins would work only when Tat is actively expressed in cells.

HIV utilizes the cellular transcriptional machinery for its own replication. Therefore, it is important to inhibit this 
step without disturbing cellular functions. Since CycT1 interacts with Tat and TAR, it can be an excellent target to develop safe and effective anti-HIV therapies. Here we present an example of a dominant negative CycT1 molecule which specifically blocks HIV transcription. Studying the precise mechanism by which this mutant CycT1 protein inhibits HIV transcription could unveil novel regulatory pathways of the HIV life cycle and therefore provide reliable clues for designing anti-HIV agents.

\section{Conclusion}

In this study, we constructed and evaluated dominant negative CycT1 mutant proteins that specifically block HIV transcription by promoting a rapid degradation of Tat proteins. These mutant CycT1 proteins represent a novel class of specific inhibitors for HIV transcription, which can be further utilized to develop a safe and effective antiHIV therapy.

\section{Methods \\ Materials}

HeLa, 293T or NIH 3T3 cells were maintained in Dulbecco's Modified Eagle's Medium (DMEM) including $10 \%$ fetal bovine serum at $37^{\circ} \mathrm{C}$ with $5 \% \mathrm{CO}_{2}$. HeLa cells stably carrying an HIV-LTR-driven luciferase reporter gene (HeLa-HR-Luc cells) were established using pHR lentiviral vector expressing the luciferase gene under the control of the HIV-LTR, as described previously $[42,43]$. Anti-myc, anti-HA, anti-CycT1, anti-Cdk9, and anti-Ub antibodies were purchased from Santa Cruz Biotechnology (Santa Cruz, CA). Anti-actin antibody was purchased from Cell Signaling Technology (Danvers, MA). Anti-Tubulin was purchased from Sigma Aldrich (St. Louis, MO). Proteasome inhibitors, MG-132 and Epoxomicin were purchased from EMD Bioscience (San Diego, CA) and Alexis (San Diego, CA), respectively.

\section{Construction of CycTI mutants}

A structure-based sequence alignment resulting from the crystal structure of the cyclin box repeat of human CycT1 [18] revealed highly conserved $\alpha$-helical structures in the P-TEFb-forming cyclins T1, T2 and K (Figure. 1B). Based on this alignment, we selected the nine most conserved regions in the cyclin box repeat domain of CycT1 and introduced random mutations into a C-terminal truncation mutant of CycT1 (1-280) by using oligonucleotides that contain degenerated nucleotides at positions corresponding to each conserved helix and the Transformer Site Directed Mutagenesis Kit (Clontech) (Figure. 1 and Table 1). The resulting 115 CycT1 mutants were tested for their ability to support Tat transactivation in murine cells as described previously [4]. The CycT1 mutants that failed to activate HIV-transcription in murine cells were sequenced and further tested for their ability to block Tat transactivation in HeLa cells as described previously [30].
The mutant CycT1 (termed CycT1-U7) that exhibited the strongest inhibitory effect on Tat-dependent HIV transcription was used in this study. Sequences of the mutagenic oligonucleotides are shown in Additional file 1.

\section{Generation of stable cell lines}

CycT-U7 was subcloned downstream of a CMV promoter in a modified pHR'-SIN lentiviral vector $[44,45]$. The VSV$\mathrm{G}$ pseudotyped lentiviruses were produced by co-transfection with packaging plasmids (pMDG and p8.9I, [46]), and used to infect Hela cells and HeLa-HR Luc cells.

\section{Transfection and reporter assays}

HeLa or NIH 3 T3 cells were transfected with $0.5 \mu \mathrm{g}$ of pEFCycT1 (wt or mutant constructs) and an HIV-Luciferase reporter construct, in the presence or absence of pTat $(0.01 \mu \mathrm{g})$ using Lipofectamine 2000 according to the manufacturer's instructions (Invitrogen). Twenty-four hours after transfection, cells were harvested and lysed. The protein concentrations of the cell lysates were determined by Protein Assay kit (BioRad). Luciferase activities in the cell lysates were measured as described previously [43].

\section{Ubiquitination assays}

HeLa cells stably expressing myc-epitope tagged mutant CycT1 proteins were expressed and, when indicated, treated with $50 \mu \mathrm{M}$ Epoxomicin for 1 hour. Cells were lysed in radio-immunoprecipitation assay (RIPA) buffer (50 mM Tris- $\mathrm{HCl}, 0.15 \mathrm{M} \mathrm{NaCl}, 1 \mathrm{mM}$ EDTA, 1\% Sodium deoxycholate, $1 \%$ NP-40, 0.1\% SDS, 1 mM DTT [pH 7.4]) in the presence of protease inhibitors. After preclearing with protein-G sepharose coupled with normal mouse IgG, cell lysates were incubated with $0.5 \mu \mathrm{g}$ of monoclonal antibody against c-Myc (F-7; Santa Cruz Biotechnology) overnight at $4{ }^{\circ} \mathrm{C}$. After the cell lysates were allowed to bind to the antibody, reaction mixtures were incubated with protein-G sepharose beads (Roche) for 1 hour at $4{ }^{\circ} \mathrm{C}$. The beads were washed extensively with RIPA buffer and the proteins remaining on the beads were eluted by incubation with SDS loading buffer (50 mM Tris-HCl, 2\% SDS, $10 \%$ glycerol, $2 \mathrm{mM}$ EDTA, $0.1 \mathrm{M}$ DTT and $0.01 \%$ bromophenol blue, pH 6.8) and subjected to SDS-PAGE, followed by Western blotting with anti-Ub antibody (Santa Cruz Biotechnology).

\section{Proteasome inhibitor treatment}

293T cells $\left(2 \times 10^{5}\right)$ were transfected with $1 \mu \mathrm{g}$ of plasmids encoding HA-tagged wt CycT1 $(1-280)$ or CycT1-U7 in the presence or absence of the plasmid encoding HAtagged HIV-1 Tat $(0.2 \mu \mathrm{g})$ using calcium phosphate. Twenty-four hours post-transfection, cells were treated with MG-132 $(50 \mu \mathrm{M})$, Epoxomicin $(50 \mu \mathrm{M})$ or DMSO (solvent control) at $37^{\circ} \mathrm{C}$ for 1,3 and 5 hours. Cells were then harvested and lysed in RIPA buffer. The protein con- 
centrations in the cell lysates were determined by Protein Assay Kits (BioRad, Palo Alto, CA). The same amounts of cellular proteins $(20 \mu \mathrm{g})$ were separated by SDS-PAGE followed by Western blot analysis to detect the HA-epitope tagged CycT1 proteins and myc-epitope tagged Tat proteins.

\section{Immunofluorescence (IF) assay}

HA-tagged wt and mutant CycT1 and myc-tagged Tat proteins were co-expressed in HeLa/HR-Luc cells using Lipofectin (Invitrogen). Twenty-four hours after transfection, cells were untreated or treated with $25 \mu \mathrm{M}$ Epoxomicin for 3 hours. Cells were fixed with $4 \%$ formaldehyde and blocked with phosphate-buffered saline (PBS) containing $0.1 \%$ Triton X-100, $1 \%$ sodium azide and $10 \%$ normal donkey serum. After washing with PBS, HA-CycT1 and were probed with mouse anti-HA monoclonal antibody (Santa Cruz) and Cy2-conjugated donkey anti-mouse IgG (Jackson ImmunoResearch). Myc-Tat proteins were probed with anti-Myc monoclonal antibody (Santa Cruz) pre-labeled with Zennon ${ }^{\circledR}$ Texas Red ${ }^{\circledR}$ anti-Mouse IgG1 (Invitrogen). Nuclei were stained with Hoechst (Sigma). Fixed cell images were captured on a Deltavision DV-RT (Applied Precision, Inc. Issaquah, WA.) microscopy system using the Deltavision Softworx program.

\section{Competing interests}

The authors declare that they have no competing interests.

\section{Authors' contributions}

JKJ carried out luciferase assays and protein assays, participated in designing the experiments and drafted the manuscript. NM constructed the mutant proteins. AS participated in the structural analysis of CycT1 protein. MG participated in sequence alignment and designing the mutant protein. TO participated in the prediction of mutant protein structure. KF participated in designing the experiments, performed biochemical experiments and helped to draft the manuscript. All authors read and approved the final manuscript.

\section{Additional material}

\section{Additional file 1}

Sequences of the mutagenic oligonucleotides.

Click here for file

[http://www.biomedcentral.com/content/supplementary/17424690-5-63-S1.doc]

\section{Acknowledgements}

We thank Keith Olszens, Lindsey McGowen, Adam Heath, Satsumi Roos, Laura Paszkowsky, Michael Zane, Renee Devor, and Yehong Huang for technical assistance, Drs. Mudit Tyagi and Monica Montano for reagents, Antonia Fraser Fujinaga for proofreading the manuscripts, and Drs.
Jonathan Karn, Matija Peterlin, Eric Arts, Catherine Patterson, Erik Andrulis, David McDonald, Ran Taube and members of Fujinaga lab for helpful discussions.

This work was supported by NIH R2I Al625I6 (KF) and Cell and Molecular Biology training grant (5TG32 GM-08056-24) (JKJ) from NIH, American Foundation of AIDS Research (AmFAR) 106386-33-RGGN (KF), the Deutsche Forschungsgemeinschaft (GE 976/5, MG), Japan Human Science Program (TO), and the Center for AIDS Research (CFAR) at Case Western Reserve University.

\section{References}

I. Karn J: Tackling Tat. J Mol Biol 1999, 293:235-254.

2. Taube R, Fujinaga K, Wimmer J, Barboric M, Peterlin BM: Tat transactivation: a model for the regulation of eukaryotic transcriptional elongation. Virology 1999, 264:245-253.

3. Bieniasz PD, Grdina TA, Bogerd HP, Cullen BR: Recruitment of a protein complex containing Tat and cyclin TI to TAR governs the species specificity of HIV-I Tat. Embo J 1998, I 7:7056-7065.

4. Fujinaga K, Taube R, Wimmer J, Cujec TP, Peterlin BM: Interactions between human cyclin $\mathrm{T}$, Tat, and the transactivation response element (TAR) are disrupted by a cysteine to tyrosine substitution found in mouse cyclin T. Proc Natl Acad Sci USA 1999, 96:1285-1290.

5. Garber ME, Wei P, KewalRamani VN, Mayall TP, Herrmann CH, Rice AP, Littman DR, Jones KA: The interaction between HIV-I Tat and human cyclin $\mathrm{TI}$ requires zinc and a critical cysteine residue that is not conserved in the murine CycTl protein. Genes Dev 1998, I 2:35 I 2-3527.

6. Kim YK, Bourgeois CF, Isel C, Churcher MJ, Karn J: Phosphorylation of the RNA polymerase II carboxyl-terminal domain by CDK9 is directly responsible for human immunodeficiency virus type I Tat-activated transcriptional elongation. Mol Cell Biol 2002, 22:4622-4637.

7. Peng J, Zhu Y, Milton JT, Price DH: Identification of multiple cyclin subunits of human P-TEFb. Genes Dev 1998, 12:755-762.

8. Kwak YT, Ivanov D, Guo J, Nee E, Gaynor RB: Role of the human and murine cyclin $T$ proteins in regulating HIV-I tat-activation. J Mol Biol 1999, 288:57-69.

9. Ivanov D, Kwak YT, Nee E, Guo J, Garcia-Martinez LF, Gaynor RB: Cyclin TI domains involved in complex formation with Tat and TAR RNA are critical for tat-activation. J Mol Biol I999, 288:4I-56.

10. Zhang J, Tamilarasu N, Hwang S, Garber ME, Huq I, Jones KA, Rana TM: HIV-I TAR RNA enhances the interaction between Tat and cyclin TI. J Biol Chem 2000, 275:343 I4-343I9.

II. Zhou Q, Yik JH: The Yin and Yang of P-TEFb regulation: implications for human immunodeficiency virus gene expression and global control of cell growth and differentiation. Microbiol Mol Biol Rev 2006, 70:646-659.

12. Michels AA, Fraldi A, Li Q, Adamson TE, Bonnet F, Nguyen VT, Sedore SC, Price JP, Price DH, Lania L, Bensaude O: Binding of the 7SK snRNA turns the HEXIMI protein into a P-TEFb (CDK9/cyclin T) inhibitor. Embo J 2004, 23:2608-26I9.

13. Michels AA, Nguyen VT, Fraldi A, Labas V, Edwards M, Bonnet $F$, Lania L, Bensaude O: MAQI and 7SK RNA interact with CDK9I cyclin $\mathbf{T}$ complexes in a transcription-dependent manner. Mol Cell Biol 2003, 23:4859-4869.

14. Barboric M, Yik JH, Czudnochowski N, Yang Z, Chen R, Contreras X, Geyer M, Matija Peterlin B, Zhou Q: Tat competes with HEXIMI to increase the active pool of P-TEFb for HIV-I transcription. Nucleic Acids Res 2007, 35:2003-20I2.

15. Schulte A, Czudnochowski N, Barboric M, Schonichen A, Blazek D, Peterlin BM, Geyer M: Identification of a cyclin T-binding domain in Hexim I and biochemical analysis of its binding competition with HIV-I Tat. J Biol Chem 2005, 280:24968-24977.

16. Bieniasz PD, Grdina TA, Bogerd HP, Cullen BR: Recruitment of cyclin TI/P-TEFb to an HIV type I long terminal repeat promoter proximal RNA target is both necessary and sufficient for full activation of transcription. Proc Natl Acad Sci USA 1999, 96:779|-7796. 
17. Garber ME, Wei P, Jones KA: HIV-I Tat interacts with cyclin TI to direct the P-TEFb CTD kinase complex to TAR RNA. Cold Spring Harb Symp Quant Biol 1998, 63:37I-380.

18. Anand K, Schulte A, Fujinaga K, Scheffzek K, Geyer M: Cyclin box structure of the P-TEFb subunit cyclin TI derived from a fusion complex with EIAV tat. J Mol Biol 2007, 370:826-836.

19. Dames SA, Schonichen A, Schulte A, Barboric M, Peterlin BM, Grzesiek S, Geyer M: Structure of the Cyclin T binding domain of Hexim I and molecular basis for its recognition of P-TEFb. Proc Natl Acad Sci USA 2007, 104: 143 I2- I43 I7.

20. Mancebo HS, Lee G, Flygare J, Tomassini J, Luu P, Zhu Y, Peng J, Blau C. Hazuda D, Price D, Flores O: P-TEFb kinase is required for HIV Tat transcriptional activation in vivo and in vitro. Genes Dev 1997, I I:2633-2644.

21. Mischiati C, Jeang KT, Feriotto G, Breda L, Borgatti M, Bianchi N, Gambari R: Aromatic polyamidines inhibiting the Tat-induced HIV-I transcription recognize structured TAR-RNA. Antisense Nucleic Acid Drug Dev 200I, I I:209-2I 7

22. Lind KE, Du Z, Fujinaga K, Peterlin BM, James TL: Structure-based computational database screening, in vitro assay, and NMR assessment of compounds that target TAR RNA. Chem Biol 2002, 9: 185-193.

23. Chao SH, Fujinaga K, Marion JE, Taube R, Sausville EA, Senderowicz AM, Peterlin BM, Price DH: Flavopiridol inhibits P-TEFb and blocks HIV-I replication. J Biol Chem 2000, 275:28345-28348.

24. Richter SN, Palu G: Inhibitors of HIV-I Tat-mediated transactivation. Curr Med Chem 2006, I 3:1305-13/5.

25. Heredia A, Davis C, Bamba D, Le N, Gwarzo MY, Sadowska M, Gallo RC, Redfield RR: Indirubin-3'-monoxime, a derivative of a Chinese antileukemia medicine, inhibits P-TEFb function and HIV-I replication. Aids 2005, 19:2087-2095

26. Hwang S, Tamilarasu N, Kibler K, Cao H, Ali A, Ping YH, Jeang KT, Rana TM: Discovery of a small molecule Tat-trans-activationresponsive RNA antagonist that potently inhibits human immunodeficiency virus-I replication. I Biol Chem 2003, 278:39092-39103

27. Okamoto H, Cujec TP, Okamoto M, Peterlin BM, Baba M, Okamoto $\mathrm{T}$ : Inhibition of the RNA-dependent transactivation and replication of human immunodeficiency virus type I by a fluoroquinoline derivative K-37. Virology 2000, 272:402-408.

28. Yedavalli VS, Benkirane M, Jeang KT: Tat and trans-activationresponsive (TAR) RNA-independent induction of HIV-I long terminal repeat by human and murine cyclin $\mathrm{TI}$ requires Sp I. J Biol Chem 2003, 278:6404-64I0.

29. Napolitano G, Mazzocco A, Fraldi A, Majello B, Lania L: Functional inactivation of Cdk9 through oligomerization chain reaction. Oncogene 2003, 22:4882-4888.

30. Fujinaga K, Irwin D, Geyer M, Peterlin BM: Optimized chimeras between kinase-inactive mutant Cdk9 and truncated cyclin TI proteins efficiently inhibit Tat transactivation and human immunodeficiency virus gene expression. J Virol 2002, 76:10873-1088I.

31. Hoque M, Tian B, Mathews MB, Pe'ery T: Granulin and granulin repeats interact with the Tat.P-TEFb complex and inhibit Tat transactivation. J Biol Chem 2005, 280:। 3648-13657.

32. Hoque M, Young TM, Lee CG, Serrero G, Mathews MB, Pe'ery T: The growth factor granulin interacts with cyclin $\mathrm{TI}$ and modulates P-TEFb-dependent transcription. Mol Cell Biol 2003, 23: $1688-1702$

33. Young TM, Wang Q, Pe'ery T, Mathews MB: The human I-mfa domain-containing protein, $\mathrm{HIC}$, interacts with cyclin $\mathrm{TI}$ and modulates P-TEFb-dependent transcription. Mol Cell Biol 2003, 23:6373-6384.

34. Bai J, Sui J, Zhu RY, Tallarico AS, Gennari F, Zhang D, Marasco WA Inhibition of Tat-mediated transactivation and HIV-I replication by human anti-hCyclinTI intrabodies. J Biol Chem 2003, 278: $1433-1442$.

35. Chao SH, Price DH: Flavopiridol inactivates $\mathbf{P}-\mathbf{T E F b}$ and blocks most RNA polymerase II transcription in vivo. I Biol Chem 200I, 276:3I793-3I799.

36. Wei P, Garber ME, Fang SM, Fischer WH, Jones KA: A novel CDK9associated C-type cyclin interacts directly with HIV-I Tat and mediates its high-affinity, loop-specific binding to TAR RNA. Cell 1998, 92:45|-462.
37. Kurosu T, Peterlin BM: VPI6 and ubiquitin; binding of P-TEFb via its activation domain and ubiquitin facilitates elongation of transcription of target genes. Curr Biol 2004, I 4: I I I 2- I I I6.

38. Fujinaga K, Irwin D, Taube R, Zhang F, Geyer M, Peterlin BM: A minimal chimera of human cyclin TI and tat binds TAR and activates human immunodeficiency virus transcription in murine cells. / Virol 2002, 76: I2934- 12939.

39. Taube R, Fujinaga K, Irwin D, Wimmer J, Geyer M, Peterlin BM: Interactions between equine cyclin TI, Tat, and TAR are disrupted by a leucine-to-valine substitution found in human cyclin TI. J Virol 2000, 74:892-898.

40. Garriga J, Grana X: Cellular control of gene expression by $\mathbf{T}$ type cyclin/CDK9 complexes. Gene 2004, 337:15-23.

4I. Peterlin BM, Price DH: Controlling the elongation phase of transcription with P-TEFb. Mol Cell 2006, 23:297-305.

42. Kim YK, Bourgeois CF, Pearson R, Tyagi M, West MJ, Wong J, Wu SY, Chiang CM, Karn ]: Recruitment of TFIIH to the HIV LTR is a rate-limiting step in the emergence of HIV from latency. Embo J 2006, 25:3596-3604.

43. Fujinaga $K$, Irwin $D$, Huang $Y$, Taube R, Kurosu T, Peterlin BM: Dynamics of Human Immunodeficiency Virus Transcription: P-TEFb Phosphorylates RD and Dissociates Negative Effectors from the Transactivation Response Element. Mol Cell Biol 2004, 24:787-795.

44. Dull T, Zufferey R, Kelly M, Mandel RJ, Nguyen M, Trono D, Naldini $L:$ A third-generation lentivirus vector with a conditional packaging system. J Virol 1998, 72:8463-847I.

45. Tyagi M, Karn J: CBF-I promotes transcriptional silencing during the establishment of HIV-I latency. EMBO J 2007, 26:4985-4995.

46. Naldini L, Blomer U, Gallay P, Ory D, Mulligan R, Gage FH, Verma IM, Trono $D$ : In vivo gene delivery and stable transduction of nondividing cells by a lentiviral vector. Science 1996, 272:263-267.

Publish with Bio Med Central and every scientist can read your work free of charge

"BioMed Central will be the most significant development for disseminating the results of biomedical research in our lifetime. "

Sir Paul Nurse, Cancer Research UK

Your research papers will be:

- available free of charge to the entire biomedical community

- peer reviewed and published immediately upon acceptance

- cited in PubMed and archived on PubMed Central

- yours - you keep the copyright
BioMedcentral 